\title{
Coupled common fixed point theorems for mixed weakly monotone mappings in partially ordered metric spaces
}

\author{
Madjid Eshaghi Gordji ${ }^{1 *}$, Esmat Akbartabar ${ }^{1}$, Yeol Je Cho ${ }^{2^{*}}$ and Maryam Ramezani ${ }^{1}$
}

\author{
* Correspondence: madjid. \\ eshaghi@gmail.com; yjcho@gnu.ac. \\ $\mathrm{kr}$ \\ ${ }^{1}$ Department of Mathematics, \\ Semnan University P.O. Box 35195- \\ 363, Semnan, Iran \\ ${ }^{2}$ Department of Mathematics \\ Education and the RINS \\ Gyeongsang National University \\ Chinju 660-701, Korea \\ Full list of author information is \\ available at the end of the article
}

\begin{abstract}
In this paper, we introduce the concept of a mixed weakly monotone pair of mappings and prove some coupled common fixed point theorems for a contractivetype mappings with the mixed weakly monotone property in partially ordered metric spaces. Our results are generalizations of the main results of Bhaskar and Lakshmikantham and Kadelburg et al.

Mathematics Subject Classification 2000: 54H25.

Keywords: common fixed point, mixed weakly monotone mappings, partially ordered metric space.
\end{abstract}

\section{Introduction}

In 1922, Banach gave a theorem, which is well-known as Banach's Fixed Point Theorem (or Banach's Contractive Principle) to establish the existence of solutions for nonlinear operator equations and integral equations. Since then, because of their simplicity and usefulness, it has become a very popular tools in solving the existence problems in many branches of mathematical analysis. Since then, many authors have extended, improved and generalized Banach's theorem in several ways [1-11].

Recently, the existence of coupled fixed points for some kinds of contractive-type mappings in partially ordered metric spaces, (ordered) cone metric spaces, fuzzy metric spaces and other spaces with applications has been investigated by some authors, for example, Bhaskar and Lakshmikantham [5], Cho et al. [12-14], Dhage et al. [15], Gordji et al. [16,17], Kadelburg et al. [18], Nieto and Lopez [10], Ran and Rarings [11], Sintunavarat et al. [19,20], Yang et al. [21] and others.

Especially, in [5], Bhaskar and Lakshmikantham introduced the notions of a mixed monotone mapping and a coupled fixed point and proved some coupled fixed point theorems for mixed monotone mappings and discussed the existence and uniqueness of solution for periodic boundary value problems.

Definition 1.1. [5] Let $(X, \leq)$ be a partially ordered set and $f: X \times X \rightarrow X$ be a mapping. We say that $f$ has the mixed monotone property on $X$ if, for any $x, y \in X$,

$$
x_{1}, x_{2} \in X, x_{1} \leq x_{2} \Rightarrow f\left(x_{1}, y\right) \leq f\left(x_{2}, y\right)
$$


and

$$
y_{1}, y_{2} \in X, y_{1} \leq y_{2} \Rightarrow f\left(x, y_{1}\right) \geq f\left(x, y_{2}\right)
$$

Definition 1.2. [5] An element $(x, y) \in X \times X$ is called a coupled fixed point of a mapping $F: X \times X \rightarrow X$ if $x=F(x, y)$ and $y=F(y, x)$.

Theorem 1.3. [5] Let $(X, \leq, d)$ be a partially ordered complete metric space. Let $f: X \times$ $X \rightarrow X$ be a mapping having the mixed monotone property on $X$. Assume that there exists $k \in[0,1)$ with

$$
\mathrm{d}(f(x, y), f(u, v)) \leq \frac{k}{2}(d(x, u)+\mathrm{d}(y, v))
$$

for all $x, y, u, v \in X$ with $x \leq u$ and $y \geq v$. Also, suppose that either

(1) $f$ is continuous or

(2) $X$ has the following properties:

(a) if $\left\{x_{n}\right\}$ is an increasing sequence with $x_{n} \rightarrow x$, then $x_{n} \leq x$ for all $n \geq 1$;

(b) if $\left\{y_{n}\right\}$ is a decreasing sequence $y_{n} \rightarrow y$, then $y_{n} \geq y$ for all $n \geq 1$.

If there exist $x_{0}, y_{0} \in X$ such that $x_{0} \leq f\left(x_{0}, y_{0}\right)$ and $y_{0} \geq f\left(y_{0}, x_{0}\right)$, then $f$ has a coupled fixed point in $X$.

Very recently, Kadelburg et al. [18] proved the following theorem on cone metric spaces.

Theorem 1.4. [18] Let $(X, \leq, d)$ be an ordered cone metric space. Let $(f, g)$ be a weakly increasing pair of self-mappings on $X$ with respect to $\leq$. Suppose that the following conditions hold:

(1) there exist $p, q, r, s, t \geq 0$ satisfying $p+q+r+s+t<1$ and $q=r$ or $s=t$ such that

$$
\mathrm{d}(f x, g y) \leq p \mathrm{~d}(x, y)+q \mathrm{~d}(x, f x)+s \mathrm{~d}(x, g y)+t \mathrm{~d}(y, f x)
$$

for all comparable $x, y \in X$;

(2) $f$ or $g$ is continuous or, if a nondecreasing $\left\{x_{n}\right\}$ converges to a point $x \in X$, then $x_{n}$ $\leq x$ for all $n \geq 1$.

Then $f$ and $g$ have a common fixed point in $X$.

Note that a pair $(f, g)$ of self-mappings on partially ordered set $(X, \leq)$ is said to be weakly increasing if $f x \leq g f x$ and $g x \leq f g x$ for all $x \in X$.

Now, we introduce the following concept of the mixed weakly increasing property of mappings.

Definition 1.5. Let $(X, \leq)$ be a partially ordered set and $f, g: X \times X \rightarrow X$ be mappings. We say that a pair $(f, g)$ has the mixed weakly monotone property on $X$ if, for any $x, y \in X$,

$$
\begin{aligned}
x & \leq f(x, y), y \geq f(y, x) \\
& \Rightarrow f(x, y) \leq g(f(x, y), f(y, x)), f(y, x) \geq g(f(y, x), f(x, y))
\end{aligned}
$$

and

$$
\begin{aligned}
& x \leq g(x, y), y \geq g(y, x) \\
& \Rightarrow g(x, y) \leq f(g(x, y), g(y, x)), g(y, x) \geq f(g(y, x), g(x, y)) .
\end{aligned}
$$


Example 1.6. Consider an ordered cone metric space $(\mathbb{R}, \leq, d)$, where $\leq$ represents the usual order relation and $d$ is a usual metric on $\mathbb{R}$ and let $f, g: \mathbb{R} \times \mathbb{R} \rightarrow \mathbb{R}$ be two functions defined by

$$
f(x, y)=x-2 y, \quad g(x, y)=x-y .
$$

Then a pair $(f, g)$ has the mixed weakly monotone property.

Example 1.7. Consider an ordered cone metric space $(\mathbb{R}, \leq, d)$, where $\leq$ represents the usual order relation and $d$ is a usual metric on $\mathbb{R}$ and let $f, g: \mathbb{R} \times \mathbb{R} \rightarrow \mathbb{R}$ be two functions defined by

$$
f(x, y)=x-y+1, \quad g(x, y)=2 x-3 y .
$$

Then both mappings $f$ and $g$ have the mixed monotone property, but a pair $(f, g)$ has not the mixed weakly monotone property. To see this, for any $\left(\frac{9}{8}, \frac{7}{8}\right) \in \mathbb{R}^{2}$, we have

$$
\frac{9}{8} \leq f\left(\frac{9}{8}, \frac{7}{8}\right), \quad \frac{7}{8} \geq f\left(\frac{7}{8}, \frac{9}{8}\right),
$$

but

$$
f\left(\frac{9}{8}, \frac{7}{8}\right) \not g\left(f\left(\frac{9}{8}, \frac{7}{8}\right), f\left(\frac{7}{8}, \frac{9}{8}\right)\right), \quad f\left(\frac{7}{8}, \frac{9}{8}\right) \geq g\left(f\left(\frac{7}{8}, \frac{9}{8}\right), f\left(\frac{9}{8}, \frac{7}{8}\right)\right) .
$$

The purpose of this paper is to present some coupled common fixed point theorems for a pair of mappings with the mixed weakly monotone property in a partially ordered metric space. Our results generalize the main results of Bhaskar and Lakshmikantham [5], Kadelburg et al. [18] and others.

\section{Coupled common fixed point theorems}

Let $(X, \leq, d)$ be a partially ordered complete metric space. Now, we consider the product space $X \times X$ with following partial order: for all $(x, y),(u, v) \in X \times X$,

$$
(x, y) \leq(u, v) \Leftrightarrow x \leq u, y \geq v .
$$

Also, let $(X \times X, D)$ be a metric space with the following metric:

$$
D((x, y),(u, v)):=\mathrm{d}(x, u)+\mathrm{d}(y, v)
$$

for all $(x, y),(u, v) \in X \times X$.

Theorem 2.1. Let $(X, \leq, d)$ be a partially ordered complete metric space. Let $f, g: X \times$ $X \rightarrow X$ be the mappings such that a pair $(f, g)$ has the mixed weakly monotone property on $X$. Suppose that there exist $p, q, r, s \geq 0$ with $p+q+r+2 s<1$ such that

$$
\begin{aligned}
d(f(x, y), g(u, v)) & \leq \frac{p}{2} D((x, y),(u, v))+\frac{q}{2} D((x, y),(f(x, y), f(y, x))) \\
& +\frac{r}{2} D((u, v),(g(u, v), g(v, u)))+\frac{s}{2} D((x, y),(g(u, v), g(v, u))) \\
& +\frac{s}{2} D((u, v),(f(x, y), f(y, x)))
\end{aligned}
$$

for all $x, y, u, v \in X$ with $x \leq u$ and $y \geq v$. Let $x_{0}, y_{0} \in X$ be such that $x_{0} \leq f\left(x_{0}, y_{0}\right)$, $y_{0} \geq f\left(y_{0}, x_{0}\right)$ or $x_{0} \leq g\left(x_{0}, y_{0}\right), y_{0} \geq g\left(y_{0}, x_{0}\right)$. If $f$ or $g$ is continuous, then $f$ and $g$ have $a$ coupled common fixed point in $X$. 
Proof. Suppose that $x_{0} \leq f\left(x_{0}, y_{0}\right)$ and $y_{0} \geq f\left(y_{0}, x_{0}\right)$ and let

$$
f\left(x_{0}, y_{0}\right)=x_{1}, \quad f\left(y_{0}, x_{0}\right)=y_{1} .
$$

From the mixed weakly monotone property of the pair $(f, g)$, we have

$$
x_{1}=f\left(x_{0}, y_{0}\right) \leq g\left(f\left(x_{0}, y_{0}\right), \quad f\left(y_{0}, x_{0}\right)\right)=g\left(x_{1}, y_{1}\right)
$$

and

$$
y_{1}=f\left(y_{0}, x_{0}\right) \geq g\left(f\left(y_{0}, x_{0}\right), \quad f\left(x_{0}, y_{0}\right)\right)=g\left(y_{1}, x_{1}\right) .
$$

Let

$$
g\left(x_{1}, y_{1}\right)=x_{2}, \quad g\left(y_{1}, x_{1}\right)=y_{2} .
$$

Then we have

$$
g\left(x_{1}, y_{1}\right) \leq f\left(g\left(x_{1}, y_{1}\right), \quad g\left(y_{1}, x_{1}\right)\right)=f\left(x_{2}, y_{2}\right)
$$

and

$$
g\left(y_{1}, x_{1}\right) \geq f\left(g\left(y_{1}, x_{1}\right), \quad g\left(x_{1}, y_{1}\right)\right)=f\left(y_{2}, x_{2}\right) .
$$

Continuously, let

$$
x_{2 n+1}=f\left(x_{2 n}, y_{2 n}\right), \quad y_{2 n+1}=f\left(y_{2 n}, x_{2 n}\right)
$$

and

$$
x_{2 n+2}=g\left(x_{2 n+1}, y_{2 n+1}\right), \quad y_{2 n+2}=g\left(y_{2 n+1}, x_{2 n+1}\right)
$$

for all $n \geq 1$. Then we can easily verify that

$$
x_{0} \leq x_{1} \leq x_{2} \leq \cdots \leq x_{n} \leq x_{n+1} \leq \cdots
$$

and

$$
y_{0} \geq y_{1} \geq y_{2} \geq \cdots \geq y_{n} \geq y_{n+1} \geq \cdots
$$

Similarly, from the condition $x_{0} \leq g\left(x_{0}, y_{0}\right)$ and $y_{0} \geq g\left(y_{0}, x_{0}\right)$, one can show that the sequences $\left\{x_{n}\right\}$ and $\left\{y_{n}\right\}$ are increasing and decreasing, respectively. Thus, applying (2.1), we obtain

$$
\begin{aligned}
& \mathrm{d}\left(x_{2 n+1}, x_{2 n+2}\right) \\
&=\mathrm{d}\left(f\left(x_{2 n}, y_{2 n}\right), g\left(x_{2 n+1}, y_{2 n+1}\right)\right) \\
& \leq \frac{p}{2} D\left(\left(x_{2 n}, y_{2 n}\right),\left(x_{2 n+1}, y_{2 n+1}\right)\right)+\frac{q}{2} D\left(\left(x_{2 n}, y_{2 n}\right),\left(f\left(x_{2 n}, y_{2 n}\right), f\left(y_{2 n}, x_{2 n}\right)\right)\right) \\
&+\frac{r}{2} D\left(\left(x_{2 n+1}, y_{2 n+1}\right),\left(g\left(x_{2 n+1}, y_{2 n+1}\right), g\left(y_{2 n+1}, x_{2 n+1}\right)\right)\right) \\
&+\frac{s}{2} D\left(\left(x_{2 n}, y_{2 n}\right),\left(g\left(x_{2 n+1}, y_{2 n+1}\right), g\left(y_{2 n+1}, x_{2 n+1}\right)\right)\right) \\
&+\frac{s}{2} D\left(\left(x_{2 n+1}, y_{2 n+1}\right),\left(f\left(x_{2 n}, y_{2 n}\right), f\left(y_{2 n}, x_{2 n}\right)\right)\right) \\
&= \frac{p}{2} D\left(\left(x_{2 n}, y_{2 n}\right),\left(x_{2 n+1}, y_{2 n+1}\right)\right)+\frac{q}{2} D\left(\left(x_{2 n}, y_{2 n}\right),\left(x_{2 n+1}, y_{2 n+1}\right)\right) \\
&+\frac{r}{2} D\left(\left(x_{2 n+1}, y_{2 n+1}\right),\left(x_{2 n+2}, y_{2 n+2}\right)\right)+\frac{s}{2} D\left(\left(x_{2 n+1}, y_{2 n+1}\right),\left(x_{2 n+1}, y_{2 n+1}\right)\right) \\
&+\frac{s}{2} D\left(\left(x_{2 n}, y_{2 n}\right),\left(x_{2 n+2}, y_{2 n+2}\right)\right) \\
& \leq \frac{p+q}{2} D\left(\left(x_{2 n}, y_{2 n}\right),\left(x_{2 n+1}, y_{2 n+1}\right)\right)+\frac{r}{2} D\left(\left(x_{2 n+1}, y_{2 n+1}\right),\left(x_{2 n+2}, y_{2 n+2}\right)\right) \\
&+\frac{s}{2}\left[D\left(\left(x_{2 n}, y_{2 n}\right),\left(x_{2 n+1}, y_{2 n+1}\right)\right)+D\left(\left(x_{2 n+1}, y_{2 n+1}\right),\left(x_{2 n+2}, y_{2 n+2}\right)\right)\right] \\
&= \frac{p+q+s}{2} D\left(\left(x_{2 n}, y_{2 n}\right),\left(x_{2 n+1}, y_{2 n+1}\right)\right)+\frac{r+s}{2} D\left(\left(x_{2 n+1}, y_{2 n+1}\right),\left(x_{2 n+2}, y_{2 n+2}\right)\right) .
\end{aligned}
$$


Hence it follows that

$$
\begin{aligned}
\mathrm{d}\left(x_{2 n+1}, x_{2 n+2}\right) & \leq \frac{p+q+s}{2}\left(\mathrm{~d}\left(x_{2 n}, x_{2 n+1}\right)+\mathrm{d}\left(y_{2 n}, y_{2 n+1}\right)\right) \\
& +\frac{r+s}{2}\left(\mathrm{~d}\left(x_{2 n+1}, x_{2 n+2}\right)+\mathrm{d}\left(y_{2 n+1}, y_{2 n+2}\right)\right)
\end{aligned}
$$

for all $n \geq 1$. Similarly, we have

$$
\begin{aligned}
\mathrm{d}\left(y_{2 n+1}, y_{2 n+2}\right) & \leq \frac{p+q+s}{2}\left(\mathrm{~d}\left(y_{2 n}, y_{2 n+1}\right)+\mathrm{d}\left(x_{2 n}, x_{2 n+1}\right)\right) \\
& +\frac{r+s}{2}\left(\mathrm{~d}\left(y_{2 n+1}, y_{2 n+2}\right)+\mathrm{d}\left(x_{2 n+1}, x_{2 n+2}\right)\right)
\end{aligned}
$$

for all $n \geq 1$. Thus it follows from (2.2) and (2.3) that

$$
\mathrm{d}\left(x_{2 n+1}, x_{2 n+2}\right)+\mathrm{d}\left(y_{2 n+1}, y_{2 n+2}\right) \leq \frac{p+q+s}{1-(r+s)}\left(\left(\mathrm{d}\left(x_{2 n}, x_{2 n+1}\right)+\mathrm{d}\left(y_{2 n}, y_{2 n+1}\right)\right)\right.
$$

for all $n \geq 1$. Moreover, if we apply (2.1), then we have

$$
\begin{aligned}
& \mathrm{d}\left(x_{2 n+2}, x_{2 n+3}\right) \\
&=\mathrm{d}\left(g\left(x_{2 n+1}, y_{2 n+1}\right), f\left(x_{2 n+2}, y_{2 n+2}\right)\right) \\
& \leq \frac{p}{2} D\left(\left(x_{2 n+1}, y_{2 n+1}\right),\left(x_{2 n+2}, y_{2 n+2}\right)\right) \\
&+\frac{q}{2} D\left(\left(x_{2 n+1}, y_{2 n+1}\right),\left(g\left(x_{2 n+1}, y_{2 n+1}\right), g\left(y_{2 n+1}, x_{2 n+1}\right)\right)\right) \\
&+\frac{r}{2} D\left(\left(x_{2 n+2}, y_{2 n+2}\right),\left(f\left(x_{2 n+2}, y_{2 n+2}\right), f\left(y_{2 n+2}, x_{2 n+2}\right)\right)\right) \\
&+\frac{s}{2} D\left(\left(x_{2 n+1}, y_{2 n+1}\right),\left(f\left(x_{2 n+2}, y_{2 n+2}\right), f\left(y_{2 n+2}, x_{2 n+2}\right)\right)\right) \\
&+\frac{s}{2} D\left(\left(x_{2 n+2}, y_{2 n+2}\right),\left(g\left(x_{2 n+1}, y_{2 n+1}\right), g\left(y_{2 n+1}, x_{2 n+1}\right)\right)\right) \\
&= \frac{p}{2} D\left(\left(x_{2 n+1}, y_{2 n+1}\right),\left(x_{2 n+2}, y_{2 n+2}\right)\right)+\frac{q}{2} D\left(\left(x_{2 n+1}, y_{2 n+1}\right),\left(x_{2 n+2}, y_{2 n+2}\right)\right) \\
&+\frac{r}{2} D\left(\left(x_{2 n+2}, y_{2 n+2}\right),\left(x_{2 n+3}, y_{2 n+3}\right)\right)+\frac{s}{2} D\left(\left(x_{2 n+1}, y_{2 n+1}\right),\left(x_{2 n+3}, y_{2 n+3}\right)\right) \\
&+\frac{s}{2} D\left(\left(x_{2 n+2}, y_{2 n+2}\right),\left(x_{2 n+2}, y_{2 n+2}\right)\right) \\
& \leq \frac{p+q}{2} D\left(\left(x_{2 n+1}, y_{2 n+1}\right),\left(x_{2 n+2}, y_{2 n+2}\right)\right)+\frac{r}{2} D\left(\left(x_{2 n+2}, y_{2 n+2}\right),\left(x_{2 n+3}, y_{2 n+3}\right)\right) \\
&+\frac{s}{2}\left[D\left(\left(x_{2 n+1}, y_{2 n+1}\right),\left(x_{2 n+2}, y_{2 n+2}\right)\right)+D\left(\left(x_{2 n+2}, y_{2 n+2}\right),\left(x_{2 n+3}, y_{2 n+3}\right)\right)\right] .
\end{aligned}
$$

Hence it follows that

$$
\begin{aligned}
\mathrm{d}\left(x_{2 n+2}, x_{2 n+3}\right) & \leq \frac{p+q+s}{2}\left(\mathrm{~d}\left(x_{2 n+1}, x_{2 n+2}\right)+\mathrm{d}\left(y_{2 n+1}, y_{2 n+2}\right)\right) \\
& +\frac{r+s}{2}\left(\mathrm{~d}\left(x_{2 n+2}, x_{2 n+2}\right)+\mathrm{d}\left(y_{2 n+3}, y_{2 n+3}\right)\right)
\end{aligned}
$$

for all $n \geq 1$. Similarly, we have

$$
\begin{aligned}
\mathrm{d}\left(y_{2 n+2}, y_{2 n+3}\right) & \leq \frac{p+q+s}{2}\left(\mathrm{~d}\left(y_{2 n+1}, y_{2 n+2}\right)+\mathrm{d}\left(x_{2 n+1}, x_{2 n+2}\right)\right) \\
& +\frac{r+s}{2}\left(\mathrm{~d}\left(y_{2 n+2}, y_{2 n+2}\right)+\mathrm{d}\left(x_{2 n+3}, x_{2 n+3}\right)\right) .
\end{aligned}
$$


Thus, using (2.5) and (2.6), we have

$$
\mathrm{d}\left(x_{2 n+2}, x_{2 n+3}\right)+\mathrm{d}\left(y_{2 n+2}, y_{2 n+3}\right) \leq \frac{p+q+s}{1-(r+s)}\left(\mathrm{d}\left(x_{2 n+1}, x_{2 n+2}\right)+\mathrm{d}\left(y_{2 n+1}, y_{2 n+2}\right)\right)
$$

for all $n \geq 1$. Also, it follows from (2.4) and (2.7) that

$$
\mathrm{d}\left(x_{2 n+2}, x_{2 n+3}\right)+\mathrm{d}\left(y_{2 n+2}, y_{2 n+3}\right) \leq\left(\frac{p+q+s}{1-(r+s)}\right)^{2}\left(\mathrm{~d}\left(x_{2 n}, x_{2 n+1}\right)+\mathrm{d}\left(y_{2 n}, y_{2 n+1}\right)\right)
$$

for all $n \geq 1$. Let $A=\frac{p+q+s}{1-(r+s)}$. Then $0 \leq A<1$ and

$$
\begin{aligned}
\mathrm{d}\left(x_{2 n+1}, x_{2 n+2}\right)+\mathrm{d}\left(y_{2 n+1}, y_{2 n+2}\right) & \leq A\left(\mathrm{~d}\left(x_{2 n}, x_{2 n+1}\right)+\mathrm{d}\left(y_{2 n}, y_{2 n+1}\right)\right) \\
& \leq A^{3}\left(\mathrm{~d}\left(x_{2 n-2}, x_{2 n-1}\right)+\mathrm{d}\left(y_{2 n-2}, y_{2 n-1}\right)\right) \\
& \leq A^{5}\left(\mathrm{~d}\left(x_{2 n-4}, x_{2 n-3}\right)+\mathrm{d}\left(y_{2 n-4}, y_{2 n-3}\right)\right) \\
& \leq \cdots \\
& \leq A^{2 n+1}\left(\mathrm{~d}\left(x_{0}, x_{1}\right)+\mathrm{d}\left(y_{0}, y_{1}\right)\right)
\end{aligned}
$$

and

$$
\begin{aligned}
\mathrm{d}\left(x_{2 n+2}, x_{2 n+3}\right)+\mathrm{d}\left(y_{2 n+2}, y_{2 n+3}\right) & \leq A^{2}\left(\mathrm{~d}\left(x_{2 n}, x_{2 n+1}\right)+\mathrm{d}\left(y_{2 n}, y_{2 n+1}\right)\right) \\
& \leq A^{4}\left(\mathrm{~d}\left(x_{2 n-2}, x_{2 n-1}\right)+\mathrm{d}\left(y_{2 n-2}, y_{2 n-1}\right)\right) \\
& \leq A^{6}\left(\mathrm{~d}\left(x_{2 n-4}, x_{2 n-3}\right)+\mathrm{d}\left(y_{2 n-4}, y_{2 n-3}\right)\right) \\
& \leq \cdots \\
& \leq A^{2 n+2}\left(\mathrm{~d}\left(x_{0}, x_{1}\right)+\mathrm{d}\left(y_{0}, y_{1}\right)\right)
\end{aligned}
$$

for all $n \geq 1$. Now, for all $m, n \geq 1$ with $n \leq m$, we have

$$
\begin{aligned}
& \mathrm{d}\left(x_{2 n+1}, x_{2 m+1}\right)+\mathrm{d}\left(y_{2 n+1}, y_{2 m+1}\right) \\
& \leq\left(\mathrm{d}\left(x_{2 n+1}, x_{2 n+2}\right)+\mathrm{d}\left(y_{2 n+1}, y_{2 n+2}\right)\right)+\left(\mathrm{d}\left(x_{2 n+2}, x_{2 n+3}\right)+\mathrm{d}\left(y_{2 n+2}, y_{2 n+3}\right)\right) \\
&+\cdots \\
&+\left(\mathrm{d}\left(x_{2 m}, x_{2 m+1}\right)+\mathrm{d}\left(y_{2 m}, y_{2 m+1}\right)\right) \\
& \leq\left(A^{2 n+1}+A^{2 n+2}+\cdots+A^{2 m}\right)\left(\mathrm{d}\left(x_{0}, x_{1}\right)+\mathrm{d}\left(y_{0}, y_{1}\right)\right) \\
& \leq \frac{A^{2 n+1}}{1-A}\left(\mathrm{~d}\left(x_{0}, x_{1}\right)+\mathrm{d}\left(y_{0}, y_{1}\right)\right) .
\end{aligned}
$$

Similarly, we have

$$
\begin{aligned}
& \mathrm{d}\left(x_{2 n}, x_{2 m+1}\right)+\mathrm{d}\left(y_{2 n}, y_{2 m+1}\right) \\
& \leq\left(A^{2 n}+A^{2 n+1}+A^{2 n+2}+\cdots+A^{2 m}\right)\left(\mathrm{d}\left(x_{0}, x_{1}\right)+\mathrm{d}\left(y_{0}, y_{1}\right)\right) \\
& \leq \frac{A^{2 n}}{1-A}\left(\mathrm{~d}\left(x_{0}, x_{1}\right)+\mathrm{d}\left(y_{0}, y_{1}\right)\right) \\
& \mathrm{d}\left(x_{2 n}, x_{2 m}\right)+\mathrm{d}\left(y_{2 n}, y_{2 m}\right) \\
& \leq\left(A^{2 n}+A^{2 n+1}+A^{2 n+2}+\cdots+A^{2 m-1}\right)\left(\mathrm{d}\left(x_{0}, x_{1}\right)+\mathrm{d}\left(y_{0}, y_{1}\right)\right) \\
& \leq \frac{A^{2 n}}{1-A}\left(\mathrm{~d}\left(x_{0}, x_{1}\right)+\mathrm{d}\left(y_{0}, y_{1}\right)\right)
\end{aligned}
$$


and

$$
\begin{aligned}
& \mathrm{d}\left(x_{2 n+1}, x_{2 m}\right)+\mathrm{d}\left(y_{2 n+1}, y_{2 m}\right) \\
& \leq\left(A^{2 n+1}+A^{2 n+1}+A^{2 n+2}+\cdots+A^{2 m-1}\right)\left(\mathrm{d}\left(x_{0}, x_{1}\right)+\mathrm{d}\left(y_{0}, y_{1}\right)\right) \\
& \leq \frac{A^{2 n+1}}{1-A}\left(\mathrm{~d}\left(x_{0}, x_{1}\right)+\mathrm{d}\left(y_{0}, y_{1}\right)\right) .
\end{aligned}
$$

Hence, for all $m, n \geq 1$ with $n \leq m$, it follows that

$$
\mathrm{d}\left(x_{n}, x_{m}\right)+\mathrm{d}\left(y_{n}, y_{m}\right) \leq \frac{A^{2 n}}{1-A}\left(\mathrm{~d}\left(x_{0}, x_{1}\right)+\mathrm{d}\left(y_{0}, y_{1}\right)\right)
$$

and so, since $0 \leq A<1$, we can conclude that

$$
\mathrm{d}\left(x_{n}, x_{m}\right)+\mathrm{d}\left(y_{n}, y_{m}\right) \rightarrow 0
$$

as $n \rightarrow \infty$, which implies that $\mathrm{d}\left(x_{n}, x_{m}\right) \rightarrow 0$ and $\mathrm{d}\left(y_{n}, y_{m}\right) \rightarrow 0$ as $m, n \rightarrow \infty$. Therefore, the sequences $\left\{x_{n}\right\}$ and $\left\{y_{n}\right\}$ are Cauchy sequences in $X$. Since $(X, d)$ is a complete metric space, then there exist $x, y \in X$ such that $x_{n} \rightarrow x$ and $y_{n} \rightarrow y$ as $n \rightarrow \infty$.

Suppose that $f$ is a continuous. Then we have

$$
x=\lim _{k \rightarrow \infty} x_{2 k+1}=\lim _{k \rightarrow \infty} f\left(x_{2 k}, y_{2 k}\right)=f\left(\lim _{k \rightarrow \infty} x_{2 k}, \lim _{k \rightarrow \infty} y_{2 k}\right)=f(x, y)
$$

and

$$
y=\lim _{k \rightarrow \infty} y_{2 k+1}=\lim _{k \rightarrow \infty} f\left(y_{2 k}, x_{2 k}\right)=f\left(\lim _{k \rightarrow \infty} y_{2 k}, \lim _{k \rightarrow \infty} x 2 k\right)=f(y, x) .
$$

Taking $x=u$ and $y=v$ in (2.1), we have

$$
\begin{aligned}
& \mathrm{d}(f(x, y), g(x, y))+\mathrm{d}(f(y, x), g(y, x)) \\
& \leq \frac{p}{2} D((x, y),(x, y))+\frac{q}{2} D((x, y), f(x, y), f(y, x)) \\
&+\frac{r}{2} D((x, y), g(x, y), g(y, x))+\frac{s}{2} D((x, y), g(x, y), g(y, x)) \\
&+\frac{s}{2} D((x, y), f(x, y), f(y, x)) \frac{p}{2} D((y, x),(y, x)) \\
&+\frac{q}{2} D((y, x), f(y, x), f(x, y))+\frac{r}{2} D((y, x), g(y, x), g(x, y)) \\
&+\frac{s}{2} D((y, x), g(y, x), g(x, y))+\frac{s}{2} D((y, x), f(y, x), f(x, y)) .
\end{aligned}
$$

Hence we have

$$
\mathrm{d}(x, g(x, y))+\mathrm{d}(y, g(y, x)) \leq(r+s)(\mathrm{d}(x, g(x, y))+\mathrm{d}(y, g(y, x)))
$$

and so, since $r+s<1$, we can get that

$$
\mathrm{d}(x, g(x, y))=0, \quad \mathrm{~d}(y, g(y, x))=0 .
$$

Hence $(x, y)$ is a coupled common fixed point of $f$ and $g$.

Similarly, we can prove that $(x, y)$ is a coupled common fixed point of $f$ and $g$ when $g$ is a continuous mapping. This completes the proof.

Theorem 2.2. Let $(X, \leq, d)$ be a partially ordered complete metric space. Assume that $X$ has the following property:

(1) if $\left\{x_{n}\right\}$ is an increasing sequence with $x_{n} \rightarrow x$, then $x_{n} \leq x$ for all $n \geq 1$; 
(2) if $\left\{y_{n}\right\}$ is a decreasing sequence with $y_{n} \rightarrow y$, then $y_{n} \geq y$ for all $n \geq 1$.

Let $f, g: X \times X \rightarrow X$ be the mappings such that a pair $(f, g)$ has the mixed weakly monotone property on $X$. Also, suppose that there exist $p, q, r, s \geq 0$ with $p+q+r+2 s$ $<1$ such that

$$
\begin{aligned}
\mathrm{d}(f(x, y), g(u, v)) & \leq \frac{p}{2} D((x, y),(u, v))+\frac{q}{2} D((x, y),(f(x, y), f(y, x))) \\
& +\frac{r}{2} D((u, v),(g(u, v), g(v, u)))+\frac{s}{2} D((x, y),(g(u, v), g(v, u))) \\
& +\frac{s}{2} D((u, v),(f(x, y), f(y, x)))
\end{aligned}
$$

for all $x, y, u, v \in X$ with $x \leq u$ and $y \geq v$. If there exist $x_{0}, y_{0} \in X$ such that $x_{0} \leq f$ $\left(x_{0}, y_{0}\right), y_{0} \geq f\left(y_{0}, x_{0}\right)$ or $x_{0} \leq g\left(x_{0}, y_{0}\right), y_{0} \geq g\left(y_{0}, x_{0}\right)$, then $f$ and $g$ have a coupled common fixed point in $X$.

Proof. Following the proof of Theorem 2.1, we only have to show that

$$
f(x, y)=g(x, y)=x, \quad f(y, x)=g(y, x)=\gamma .
$$

It is clear that

$$
\begin{aligned}
& D((x, y),(f(x, y), f(y, x))) \\
& \leq D\left((x, y),\left(x_{2 k+2}, y_{2 k+2}\right)\right)+D\left(\left(x_{2 k+2}, y_{2 k+2}\right),(f(x, y), f(y, x))\right) \\
& =D\left((x, y),\left(x_{2 k+2}, y_{2 k+2}\right)\right)+D\left(\left(g\left(x_{2 k+1}, y_{2 k+1}\right), g\left(y_{2 k+1}, x_{2 k+1}\right)\right),(f(x, y), f(y, x))\right) \\
& =D\left((x, y),\left(x_{2 k+2}, y_{2 k+2}\right)\right)+\mathrm{d}\left(g\left(x_{2 k+1}, y_{2 k+1}\right), f(x, y)\right)+\mathrm{d}\left(f(y, x), g\left(y_{2 k+1}, x_{2 k+1}\right)\right) \\
& \leq D\left((x, y),\left(x_{2 k+2}, y_{2 k+2}\right)\right)+\frac{p}{2} D\left(\left(x_{2 k+1}, y_{2 k+1}\right),(x, y)\right) \\
& +\frac{q}{2} D\left(\left(x_{2 k+1}, y_{2 k+1}\right),\left(g\left(x_{2 k+1}, y_{2 k+1}\right), g\left(y_{2 k+1}, x_{2 k+1}\right)\right)\right)+\frac{r}{2} D((x, y),(f(x, y), f(y, x))) \\
& +\frac{s}{2} D\left(\left(x_{2 k+1}, \gamma_{2 k+1}\right),(f(x, y), f(y, x))\right)+\frac{s}{2} D\left((x, y),\left(g\left(x_{2 k+1}, \gamma_{2 k+1}\right), g\left(\gamma_{2 k+1}, x_{2 k+1}\right)\right)\right) \\
& +\frac{p}{2} D\left((y, x),\left(y_{2 k+1}, x_{2 k+1}\right)\right)+\frac{q}{2} D((y, x),(f(y, x), f(x, y))) \\
& +\frac{r}{2} D\left(\left(y_{2 k+1}, x_{2 k+1}\right),\left(g\left(y_{2 k+1}, x_{2 k+1}\right), g\left(x_{2 k+1}, y_{2 k+1}\right)\right)\right) \\
& +\frac{s}{2} D\left((y, x),\left(g\left(y_{2 k+1}, x_{2 k+1}\right), g\left(x_{2 k+1}, y_{2 k+1}\right)\right)\right)+\frac{s}{2} D\left(\left(y_{2 k+1}, x_{2 k+1}\right),(f(y, x), f(x, y))\right)
\end{aligned}
$$

and so

$$
\begin{aligned}
\mathrm{d}(x, & f(x, y))+\mathrm{d}(y, f(y, x)) \\
\leq & \mathrm{d}\left(x, x_{2 k+2}\right)+\mathrm{d}\left(y, y_{2 k+2}\right)+p\left(\mathrm{~d}\left(x_{2 k+1}, x\right)+\mathrm{d}\left(y_{2 k+1}, y\right)\right) \\
& +\frac{q}{2}\left(\mathrm{~d}\left(x_{2 k+1}, x_{2 k+2}\right)+\mathrm{d}\left(y_{2 k+1}, y_{2 k+2}\right)+\mathrm{d}(x, f(x, y))+\mathrm{d}(y, f(y, x))\right) \\
& +\frac{r}{2}\left(\mathrm{~d}(x, f(x, y))+\mathrm{d}(y, f(y, x))+\mathrm{d}\left(y_{2 k+1}, y_{2 k+2}\right)+\mathrm{d}\left(x_{2 k+1}, x_{2 k+2}\right)\right) \\
& +s\left(\mathrm{~d}\left(x_{2 k+2}, x\right)+\mathrm{d}\left(y_{2 k+2}, y\right)+\mathrm{d}\left(x_{2 k+1}, f(x, y)\right)+\mathrm{d}\left(y_{2 k+1}, f(y, x)\right)\right) .
\end{aligned}
$$

Letting $k \rightarrow \infty$ in (2.9), we obtain

$$
\mathrm{d}(x, f(x, y))+\mathrm{d}(y, f(y, x)) \leq \frac{q+r+2 s}{2}[\mathrm{~d}(x, f(x, y))+\mathrm{d}(y, f(y, x))] .
$$

Since $\frac{q+r+2 s}{2}<1$, we have

$$
\mathrm{d}(x, f(x, y))+\mathrm{d}(y, f(y, x))=0
$$


and so $f(x, y)=x$ and $f(y, x)=y$. Similarly, we can show that $g(x, y)=x$ and $g(y, x)=$ $y$. Therefore, $(x, y)$ is a coupled common fixed point of $f$ and $g$. This completes the proof. $\square$

Now, we give an example to illustrate Theorem 2.1 as follows:

Example 2.3. Consider $(\mathbb{R}, \leq, d)$, where $\leq$ represents the usual order relation and $d$ is a usual metric on $\mathbb{R}$ and let $f, g: \mathbb{R} \times \mathbb{R} \rightarrow \mathbb{R}$ be two functions defined by

$$
f(x, y)=\frac{6 x-3 y+33}{36}, \quad g(x, y)=\frac{8 x-4 y+44}{48} \text {. }
$$

Then a pair $(f, g)$ has the mixed weakly monotone property and

$$
\begin{aligned}
\mathrm{d}(f(x, y), g(u, v))=|f(x, y)-g(u, v)| & =\left|\frac{6 x-3 y+33}{36}-\frac{8 x-4 y+44}{48}\right| \\
& \leq \frac{1}{6}|x-u|+\frac{1}{12}|y-v| \\
& \leq \frac{1}{6}(|x-u|+|y-v|) .
\end{aligned}
$$

By putting $p=\frac{1}{3}$ and $q=r=s=0$ in $(2.1)$, we see that $(1,1)$ is a unique coupled common fixed point of $f$ and $g$.

Corollary 2.4. In Theorems 2.1 and 2.2, if $X$ is a total ordered set, then a coupled common fixed point of $f$ and $g$ is unique and $x=y$.

Proof. If $\left(x^{*}, y^{*}\right) \in X \times X$ is another coupled common fixed point of $f$ and $g$, then, by the use of (2.1), we have

$$
\begin{aligned}
\mathrm{d}(x, & \left.x^{*}\right)+\mathrm{d}\left(y, y^{*}\right) \\
= & \mathrm{d}\left(f(x, y), g\left(x^{*}, y^{*}\right)\right)+\mathrm{d}\left(f(y, x), g\left(y^{*}, x^{*}\right)\right) \\
\leq & \frac{p}{2} D\left((x, y),\left(x^{*}, y^{*}\right)\right)+\frac{q}{2} D((x, y),(f(x, y), f(y, x))) \\
& +\frac{r}{2} D\left(\left(x^{*}, y^{*}\right),\left(g\left(x^{*}, y^{*}\right), g\left(y^{*}, x^{*}\right)\right)\right)+\frac{s}{2} D\left((x, y),\left(g\left(x^{*}, y^{*}\right), g\left(y^{*}, x^{*}\right)\right)\right) \\
& +\frac{s}{2} D\left(\left(x^{*}, y^{*}\right),(f(x, y), f(y, x))\right)+\frac{p}{2} D\left((y, x),\left(y^{*}, x^{*}\right)\right) \\
& +\frac{q}{2} D((y, x),(f(y, x), f(x, y)))+\frac{r}{2} D\left(\left(y^{*}, x^{*}\right),\left(g\left(y^{*}, x^{*}\right), g\left(x^{*}, y^{*}\right)\right)\right) \\
& +\frac{s}{2} D\left((y, x),\left(g\left(y^{*}, x^{*}\right), g\left(x^{*}, y^{*}\right)\right)\right)+\frac{s}{2} D\left(\left(\left(y^{*}, x^{*}\right),(f(y, x), f(x, y))\right)\right. \\
= & \left.\left.p\left(\mathrm{~d}\left(x, x^{*}\right)\right)+\mathrm{d}\left(y, y^{*}\right)\right)+2 s\left(\mathrm{~d}\left(x, x^{*}\right)\right)+\mathrm{d}\left(y, y^{*}\right)\right)
\end{aligned}
$$

and hence

$$
\mathrm{d}\left(x, x^{*}\right)+\mathrm{d}\left(y, y^{*}\right)=(p+2 s)\left(\mathrm{d}\left(x, x^{*}\right)+\mathrm{d}\left(y, y^{*}\right)\right) .
$$

Since $q+2 s<1$, we have $\mathrm{d}\left(x, x^{*}\right)+\mathrm{d}\left(y, y^{*}\right)=0$, which implies that $x=x^{*}$ and $y=y^{*}$. On the other hand, we have

$$
\begin{aligned}
\mathrm{d}(x, y) & =\mathrm{d}(f(x, y), g(y, x)) \\
& \leq \frac{p}{2} D((x, y),(y, x))+s D((x, y),(y, x)) \\
& =(p+2 s) \mathrm{d}(x, y) .
\end{aligned}
$$

Since $p+2 s<1$, we have $\mathrm{d}(x, y)=0$ and $x=y$. This completes the proof. 
Let $f: X \times X \rightarrow X$ be a mapping. Now, we denote

$$
f^{n+1}(x, y)=f\left(f^{n}(x, y), f^{n}(y, x)\right)
$$

for all $x, y \in X$ and $n \geq 1$.

Remark 2.5. Let $(X, \leq, d)$ be a partially ordered complete metric space. Let $f: X \times X$ $\rightarrow X$ be a mapping with the mixed monotone property on $X$. Then, for each $n \geq 1$, a pair $\left(f^{n}, f^{n}\right)$ has the mixed weakly monotone property on $X$. In fact, let $x \leq f^{n}(x, y)$ and $y \leq f^{n}(y, x)$. Then it follows from the mixed monotone property of $f$ that

$$
\begin{aligned}
& f(x, y) \leq f\left(f^{n}(x, y), y\right) \leq f\left(f^{n}(x, y), \quad f^{n}(y, x)\right)=f^{n+1}(x, y), \\
& f(y, x) \geq f\left(\left(f^{n}(y, x), x\right) \geq f\left(f^{n}(y, x), \quad f^{n}(x, y)\right)=f^{n+1}(y, x)\right.
\end{aligned}
$$

and

$$
\begin{aligned}
& f^{2}(x, y)=f(f(x, y), f(y, x)) \leq f\left(f^{n+1}(x, y), \quad f^{n+1}(y, x)\right)=f^{n+2}(x, y) \\
& f^{2}(y, x)=f(f(y, x), f(x, y)) \geq f\left(f^{n+1}(y, x), \quad f^{n+1}(x, y)\right)=f^{n+2}(y, x) .
\end{aligned}
$$

Continuously, we have

$$
f^{n}(x, y) \leq f^{n+n}(x, y), \quad f^{n}(y, x) \geq f^{n+n}(y, x)
$$

Hence we have

$$
f^{n}(x, y) \leq f^{n}\left(f^{n}(x, y), f^{n}(y, x)\right), \quad f^{n}(y, x) \geq f^{n}\left(f^{n}(y, x), f^{n}(x, y)\right),
$$

which implies that the pair $\left(f^{n}, f^{n}\right)$ has the mixed weakly monotone property on $X$.

Corollary 2.6. Let $(X, \leq, d)$ be a partially ordered complete metric space. Let $f: X \times X$ $\rightarrow X$ be a mapping with the mixed monotone property on $X$. Assume that there exist $p$, $q, r, s \geq 0$ with $p+q+r+2 s<1$ such that

$$
\begin{aligned}
\mathrm{d}(f(x, y), f(u, v)) & \leq \frac{p}{2} D((x, y),(u, v))+\frac{q}{2} D((x, y),(f(x, y), f(y, x))) \\
& +\frac{r}{2} D((u, v),(f(u, v), f(v, u)))+\frac{s}{2} D((x, y),(f(u, v), f(v, u))) \\
& +\frac{s}{2} D((u, v),(f(x, y), f(y, x)))
\end{aligned}
$$

for all $x, y, u, v \in X$ with $x \leq u$ and $y \geq v$. Moreover, suppose that either

(1) $f$ is continuous or

(2) $X$ has the following properties:

(a) if $\left\{x_{n}\right\}$ is an increasing sequence with $x_{n} \rightarrow x$, then $x_{n} \leq x$ for all $n \geq 1$;

(b) if $\left\{y_{n}\right\}$ is a decreasing sequence with $y_{n} \rightarrow y$, then $y_{n} \geq y$ for all $n \geq 1$.

If there exist $x_{0}, y_{0} \in X$ such that $x_{0} \leq f\left(x_{0}, y_{0}\right)$ and $y_{0} \geq f\left(y_{0}, x_{0}\right)$, then $f$ has a coupled fixed point in $X$.

Proof. Taking $f=g$ in Theorems 2.1, 2.2 and using Remark 2.5, we can get the conclusion. $\square$

Corollary 2.7. Let $(X, \leq, d)$ be a partially ordered complete metric space. Let $f: X \times X \rightarrow$ $X$ be a mapping with the mixed monotone property on $X$. Assume that there exists $k \in[0$, 1) with 


$$
\mathrm{d}(f(x, y), f(u, v)) \leq \frac{k}{2}(\mathrm{~d}(x, u)+\mathrm{d}(y, v))
$$

for all $x, y, u, v \in X$ with $x \leq u$ and $y \geq v$. Also, suppose that either

(1) $f$ is continuous or

(2) X has the following properties:

(a) if $\left\{x_{n}\right\}$ is an increasing sequence with $x_{n} \rightarrow x$, then $x_{n} \leq x$ for all $n \geq 1$;

(b) if $\left\{y_{n}\right\}$ is a decreasing sequence with $y_{n} \rightarrow y$, then $y_{n} \geq y$ for all $n \geq 1$.

If there exist $x_{0}, y_{0} \in X$ such that $x_{0} \leq f\left(x_{0}, y_{0}\right)$ and $y_{0} \geq f\left(y_{0}, x_{0}\right)$, then $f$ has a coupled fixed point in $X$.

Proof. Taking $f=g, p=k$ and $q=r=s=0$ in Theorems 2.1, 2.2 and using Remark 2.5 , we can get the conclusion.

\section{Acknowledgements}

The authors would like to thank anonymous referees for their valuable comments and suggestions. This research was also supported by Basic Science Research Program through the National Research Foundation of Korea (NRF) funded by the Ministry of Education, Science and Technology (Grant Number: 2011-0021821).

\section{Author details}

${ }^{1}$ Department of Mathematics, Semnan University P.O. Box 35195-363, Semnan, Iran ${ }^{2}$ Department of Mathematics Education and the RINS Gyeongsang National University Chinju 660-701, Korea

\section{Authors' contributions}

All authors read and approved the final manuscript.

\section{Competing interests}

The authors declare that they have no competing interests.

Received: 26 September 2011 Accepted: 12 June 2012 Published: 12 June 2012

\section{References}

1. Agarwal, RP, O'Regan, D: Fixed point theory for admissible type maps with applications. Fixed Point Theory Appl 2009, 22 (2009). Article ID 439176

2. Agarwal, RP, Zhou, Y, He, Y: Existence of fractional neutral functional differential equations. Comput Math Appl. 59, 1095-1100 (2010). doi:10.1016/j.camwa.2009.05.010

3. Amini-Harandi, A, Farajzadeh, AP, O'Regan, D, Agarwal, RP: Coincidence point, best approximation, and best proximity theorems for condensing set-valued maps in hyperconvex metric spaces. Fixed Point Theory Appl 2008, 8 (2008). Article ID 543154

4. Ariza-Ruiz, Jimnez-Melado, A: A continuation method for weakly contractive mappings under the interior condition. Fixed Point Theory Appl 2009, 8 (2009). Article ID 809315

5. Bhaskar, TG, Lakshmikantham, V: Fixed point theorems in partially ordered metric spaces and applications. Nonlinear Anal. 65, 1379-1393 (2006). doi:10.1016/..na.2005.10.017

6. Chang, SS, Cho, YJ, Huang, NJ: Coupled fixed point theorems with applications. J Korean Math Soc. 33, 575-585 (1996)

7. Cho, YJ, He, G, Huang, NJ: The existence results of coupled quasi-solutions for a class of operator equations. Bull Korean Math Soc. 47, 455-465 (2010)

8. Guo, D, Cho, YJ, Zhu, J: Partial Ordering Methods in Nonlinear Problems. Nova Science Publishers, Inc, New York. (2004)

9. Huang, NJ, Fang, YP, Cho, YJ: Fixed point and coupled fixed point theorems for multi-valued increasing operators in ordered metric spaces. pp. 91-98. Fixed Point Theory and Applications Nova Science Publishers, Inc, New York3, (2002)

10. Nieto, JJ, Lopez, RR: Contractive mapping theorems in partially ordered sets and applications to ordinary differential equations. Order. 22, 223-239 (2005). doi:10.1007/s11083-005-9018-5

11. Ran, ACM, Reurings, MCB: A fixed point theorem in partially ordered sets and some application to matrix equations. Proc Am Math Soc. 132, 1435-1443 (2004). doi:10.1090/S0002-9939-03-07220-4

12. Cho, YJ, Rhoades, BE, Saadati, R, Samet, B, Shantawi, W: Nonlinear coupled fixed point theorems in ordered generalized metric spaces with integral type. Fixed Point Theory Appl. 2012, 8 (2012). doi:10.1186/1687-1812-2012-8

13. Cho, YJ, Saadati, R, Wang, S: fixed point theorems on generalized distance in order cone metric spaces. Comput Math Appl. 61, 1254-1260 (2011). doi:10.1016/j.camwa.2011.01.004

14. Cho, YJ, Shah, MH, Hussain, N: Coupled fixed points of weakly F-contractive mappings in topological spaces. Appl Math Lett. 24, 1185-1190 (2011). doi:10.1016/j.aml.2011.02.004

15. Dhage, BC, O'Regan, D, Agarwal, RP: Common fixed point theorems for a pair of countably condensing mappings in ordered banach spaces. J Appl Math Stoch Anal. 16, 243-248 (2003). doi:10.1155/S1048953303000182 
16. Gordji, ME, Baghani, H, Cho, YJ: Coupled fixed point theorems for contractions in intuitionistic fuzzy normed spaces. Math Comput Model. 54, 1897-1906 (2011). doi:10.1016/j.mcm.2011.04.014

17. Gordji, ME, Ramezani, M: A generalization of Mizoguchi and Takahashi's theorem for single-valued mappings in partially ordered metric spaces. Nonlinear Anal. 74, 4544-4549 (2001)

18. Kadelburg, Z, Pavlovic, M, Radenovic, S: Common fixed point theorems for ordered contractions and quasicontractions in ordered cone metric spaces. Comput Math Appl. 59, 3148-3159 (2010). doi:10.1016/j.camwa.2010.02.039

19. Sintunavarat, W, Cho, YJ, Kumam, P: Common fixed point theorems for c-distance in ordered cone metric spaces. Comput Math Appl. 62, 1969-1978 (2011). doi:10.1016/j.camwa.2011.06.040

20. Sintunavarat, W, Cho, YJ, Kumam, P: Coupled coincidence point theorems for contractions without commutative condition in intuitionistic fuzzy normed spaces. Fixed Point Theory Appl. 2011, 81 (2011). doi:10.1186/1687-1812-201181

21. Yang, SK, Bae, JS, Cho, SH: Coincidence and common fixed and periodic point theorems in cone metric spaces. Comput Math Appl. 61, 170-177 (2011). doi:10.1016/j.camwa.2010.10.031

doi:10.1186/1687-1812-2012-95

Cite this article as: Gordji et al:: Coupled common fixed point theorems for mixed weakly monotone mappings

in partially ordered metric spaces. Fixed Point Theory and Applications 2012 2012:95.

\section{Submit your manuscript to a SpringerOpen ${ }^{\circ}$} journal and benefit from:

- Convenient online submission

- Rigorous peer review

- Immediate publication on acceptance

- Open access: articles freely available online

- High visibility within the field

- Retaining the copyright to your article

Submit your next manuscript at $\gg$ springeropen.com 